\title{
Adaptación transcultural al español de los instrumentos de tamizaje auditivo para escolares TEAP y ABEL
}

\section{Transcultural adaptation to Spanish of the hearing screening questionnaires TEAP and ABEL}

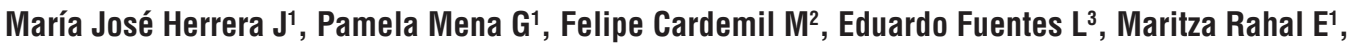 \\ David Sanhueza C ${ }^{4}$, Javiera Pardo J1, Suzanne Purdy $\mathbf{C}^{5}$.
}

\begin{abstract}
RESUMEN
Introducción: La hipoacusia es una condición prevalente que tiene consecuencias en el desarrollo de los individuos. Cada vez cobran mayor importancia los programas de tamizaje de hipoacusia. Una estrategia involucra la utilización de cuestionarios de tamizaje de hipoacusia.

Objetivos: Realizar la adaptación transcultural al español de los instrumentos de tamizaje auditivos ABEL y TEAP.

Material y método: Se realizó un estudio de corte transversal de validación de cuestionario en escolares de cuarto básico. Se utilizó la teoría clásica de tests, que incluye validez de criterio, confiabilidad test-retest y consistencia interna.

Resultados: Fueron evaluados 100 escolares de San Bernardo. Cincuenta y dos por ciento de la muestra fueron mujeres. El promedio (DE) de edad fue 9,75 $( \pm 0,78)$ años. Hubo tres casos de hipoacusia bilateral y dos de hipoacusia unilateral. La consistencia interna del test ABEL fue de 0,73. La consistencia interna del test TEAP fue de 0,82. La confiabilidad test-retest del ABEL fue 0,86 y del TEAP fue 0,86. No hubo diferencia entre los puntajes del ABEL ni del TEAP entre niños con hipoacusia y sin hipoacusia.

Conclusión: Sería de utilidad utilizar cuestionarios para el tamizaje de hipoacusia en escolares. Estos resultados permiten plantear un programa formal de tamizaje basado en cuestionarios de hipoacusia.
\end{abstract}

Palabras clave: Hipoacusia, tamizaje, escolares.

\footnotetext{
1 Servicio de Otorrinolaringología, Hospital Barros Luco Trudeau - Universidad de Chile.

2 Servicio de Otorrinolaringología, Hospital San Juan de Dios - Universidad de Chile y Depto. ORL Clínica Las Condes.

3 Facultad de Medicina, Carrera de Fonoaudiología, Pontificia Universidad Católica de Chile.

4 Médico Cirujano, Universidad de Chile.

5 SpeechScience, School of Psychology, Faculty of Science, The University of Auckland, New Zealand.
} 


\section{ABSTRACT}

Introduction: Hearinglossis a prevalent condition that has consequences for children's development. As a result, screening programs for hearing loss are of increasing importance. One screening strategy involves the use of questionnaires for people with hearing loss.

Aim: Transcultural adaptation to Spanish of the hearing screening in struments ABEL and the TEAP.

Material and method: A cross-sectional questionnaire validation study in fourth grade school children was conducted. Classical test theory, including criterion validity, test-retest reliability and internal consistency was used.

Results: A total of 100 school children in San Bernardo were evaluated. 52\% of the sample were girls. The mean (SD) age was $9.75( \pm 0.78)$ years. There were three cases of bilateral hearing loss and two cases of unilateral hearing loss. The internal consistency of the test was 0.73 for ABEL. The internal consistency was 0.82 for TEAP. The test-retest reliability was 0.86 for ABEL and for TEAP was 0.86 . There was no difference between the scores of ABEL or TEAP between the children with hearing loss and those without hearing loss.

Conclusions: It would be helpful to use questionnaires to screen for hearing loss in school. These results support a formal screening program based on questionnaires of hearing loss.

Key words: Hearing loss, screening, school children.

\section{INTRODUCCIÓN}

La hipoacusia tiene una incidencia de 1 a 4 por cada 1.000 recién nacidos vivos ${ }^{1}$, y se estima que 9 a 10 de cada mil niños desarrollan sordera en edad escolar². La prevalencia de hipoacusia aumenta con la edad, agregándose hipoacusia de conducción de cualquier causa, como tapones de cerumen, efusión timpánica u otitis media crónica, y los casos de hipoacusia sensorioneural de inicio tardío o progresivas, además de los desórdenes de procesamiento auditivo ${ }^{3}$.

En Chile, un problema de salud relevante en la edad escolar corresponde a hipoacusia. La encuesta nacional de calidad de vida y salud del año 2006 mostró que dentro de los problemas de salud reportados por los padres se encontraban problemas de audición en el 2,6\% de los encuestados ${ }^{4}$. En el año 2005 se realizó un estudio audiológico a niños de 4 y 5 años de un jardín infantil del área norte de la Región Metropolitana, preguntando además a las educadoras acerca de la sospecha de pérdida auditiva o problemas de lenguaje en los niños evaluados. La prevalencia de hipoacusia fue $11,6 \%$, correspondiendo todos los casos a hipoacusia de transmisión. La sensibilidad de las educadoras para pesquisarla fue $50 \%{ }^{5}$.

Múltiples programas de detección de hipoacusia se han implementado en el mundo. En el año 2011, en el $10^{\circ}$ Congreso de la Federación de Sociedades de Audiología europeas, expertos de la comunidad elaboraron un consenso en relación al tamizaje auditivo en escolares y preescolares. Dicho documento concluye que es de gran importancia motivar a las autoridades de los países de la comunidad europea en implementar un tamizaje de hipoacusia en este grupo de niños, para entregarle oportunidades educacionales equivalentes a los niños sanos. Este debe orientarse especialmente a niños entre 4 y 7 años, con test validados con medicina basada en evidencia internacionalmente, y con un permanente control de calidad ${ }^{3}$. En el Reino Unido existe un programa de detección precoz de hipoacusia al entrar al sistema escolar, que se realiza a niños de 4 a 5 años. La detección se realiza con un barrido audiométrico de tonos puros, con alta variabilidad en su implementación. En un estudio que analiza este tamizaje se objetiva que es un test altamente sensible y específico, con una buena detección de alteraciones auditivas, pero que tiene un costo elevado com- 
parado con otras estrategias de tamizaje, como la utilización de cuestionarios o de otoemisiones acústicas ${ }^{6}$. En nuestro país, la JUNAEB (Junta Nacional de Auxilio Escolar y Becas), en su Programa MECE (Mejoramiento en la Calidad y Equidad de la Educación) contempla un tamizado gratuito de audición a preescolares y escolares de escuelas municipales y particulares subvencionadas ${ }^{5}$, mediante la aplicación de un instrumento de evaluación de hipoacusia y la realización de audiometrías a los casos sospechosos. Sin embargo, no existen reportes formales de los resultados de este programa, principalmente respecto a su cobertura y costos. Además, han existido observaciones respecto a este programa considerando que no está disponible el resultado de la validación de éste, lo que tiene importancia en la medida que los programas de salud deberían estar basados en estrategias probadamente efectivas.

Una estrategia utilizada internacionalmente para realizar tamizaje es aplicar cuestionarios validados. En este sentido, la encuesta ABEL (Auditory Behavior in Every day Life) para padres es útil para detección de hipoacusia. Ha sido validada y aplicada con buenos resultados en población con hipoacusia neozelandesa y brasileña ${ }^{7,8}$. La misma autora de esta encuesta, basándose en encuestas antiguas, creó la encuesta para profesores TEAP (Teachers Evaluation of Auditory Performance). Esta evalúa el comportamiento auditivo en la sala de clases y permite evidenciar deficiencias auditivas en niños que padecen patología de la audición?

\section{OBJETIVO}

El objetivo del presente estudio fue realizar una adaptación transcultural al español de los instrumentos de tamizaje auditivos ABEL y TEAP en escolares y profesores de la zona sur de Santiago.

\section{MATERIAL Y MÉTODO}

Se realizó un estudio de corte transversal de validación de cuestionario en escolares de cuarto básico y en profesores de escuelas públicas de la comuna de San Bernardo durante diciembre del año 2012. El estudio contó con aprobación del Comité de Ética del Servicio de Salud Metropolitano Sur.
Cada padre o madre de los escolares, así como los profesores, firmaron un consentimiento informado para los fines del presente estudio.

Se analizaron las variables sexo, edad, otoscopía, presencia de hipoacusia, tipo de hipoacusia, bilateralidad de la hipoacusia, umbral audiométrico y puntaje obtenido en los cuestionarios ABEL y TEAP. Estos cuestionarios fueron aplicados a escolares de cuarto básico debido a que se realizó durante una instancia de rediseño del programa de salud escolar del Ministerio de Salud y realización de un nuevo piloto de este programa, que estaba enfocado en escolares de ese curso.

El instrumento ABEL es un instrumento de 24 preguntas orientado a los padres de niños en que se sospecha hipoacusia ${ }^{7}$. Consta de 3 factores: auraloral, conciencia auditiva, y habilidades sociales y de conversación. Cada pregunta pertenece a un factor, y se gradúa en una escala Likert de 0 a 6 . Una de las preguntas del cuestionario original se refiere a la mejoría luego de la implementación con implantes cocleares. Considerando que este cuestionario se propone para evaluar la percepción auditiva de los padres respecto a la audición de sus hijos, y con la aprobación de la autora original del estudio, esa pregunta se excluyó de la validación, por lo que el cuestionario que se validó contenía 23 preguntas. El instrumento TEAP está orientado a los profesores de niños con sospecha de hipoacusia ${ }^{9}$. Consta de 2 secciones: TEAP-A que tiene 4 preguntas que se gradúan en una escala Likert de +1 a -5 , y TEAP-B que tiene 6 preguntas de afirmación o negación.

Cada uno de los escolares participantes fue evaluado mediante los cuestionarios ABEL y TEAP, con otoscopía, y con audiometría tonal para determinar la presencia y el grado de hipoacusia. Para la adaptación transcultural se utilizó la teoría clásica de los tests ${ }^{10}$. En este caso en particular se realizó validación de apariencia, validez de criterio (utilizando la audiometría como gold standard), validez de constructo, confiabilidad test-retest, y consistencia interna. Para la confiabilidad test-retest se realizaron 2 aplicaciones de los cuestionarios, las que estuvieron separadas por un período de 4 semanas entre ellas. En el caso de la validación de constructo de los cuestionarios se realizó una análisis factorial exploratorio.

Para el análisis estadístico se utilizó Stata 11. Se utilizaron descriptores estadísticos generales como promedio y desviación estándar (DE) 
0 mediana y rango intercuartil (P25-P75) para variables cuantitativas, y frecuencias absolutas y relativas para variables cualitativas. En el caso del análisis factorial, y dado que los cuestionarios se graduaron en una escala tipo Likert, se utilizó una matriz de correlaciones policóricas. Para el análisis test-retest se utilizó coeficiente de correlación intraclase. Para analizar la consistencia interna se utilizó alfa de Cronbach.

\section{RESULTADOS}

Fueron evaluados 100 escolares de San Bernardo. Cincuenta y dos por ciento de la muestra fueron mujeres. El promedio (DE) de edad fue $9,75( \pm 0,78)$ años. El umbral auditivo promedio del oído derecho fue $8,38 \mathrm{~dB}$, y el del oído izquierdo $7,84 \mathrm{~dB}$.

De los 5 casos pesquisados con hipoacusia, tres casos correspondieron a hipoacusia bilateral y 2 a casos de hipoacusia unilateral. Todos los casos correspondieron a hipoacusia en rango de leve. La mediana (RIC) de umbral auditivo del oído derecho fue de 26,25 (21,25 - 30) dB y en el izquierdo fue de 21,25 (10 - 22,5) dB (Tabla 1).

La consistencia interna del test ABEL fue de 0,73 . La consistencia interna del test TEAP fue de 0,82 . La confiabilidad test-retest del ABEL fue 0,86 y del TEAP fue 0,86 . En cuanto a la estructura factorial de los cuestionarios, para el caso del test ABEL, subyacen tres factores, los que dan cuenta del $80 \%$ de la varianza $(57 \%, 13 \%$ y $10 \%$, respectivamente). Mientras que en el test TEAP, se identificó un factor para cada una de sus partes ( $A$ y $B$ ).
En cuanto a los puntajes del cuestionario ABEL se observaron diferencias entre normales e hipoacúsicos, tanto en el puntaje final como en cada uno de sus factores, sin embargo éstas no resultaron ser estadísticamente significativas (Tabla 2). Algo similar ocurrió con el cuestionario TEAP.

En los Anexos 1 y 2 se aprecian las versiones finales adaptadas al español de los cuestionarios.

\section{DISCUSIÓN}

La hipoacusia infantil tiene consecuencias conocidas en diversas áreas, dificulta el desarrollo intelectual y del lenguaje ${ }^{11}$, generando también un impacto a nivel social y emocional ${ }^{12}$. Un tamizaje universal que permita detectar niños con esta patología, evitaría las secuelas de una condición que es tratable. En Chile, a pesar de esfuerzos de

Tabla 1. Umbral auditivo de 5 escolares con hipoacusia

\begin{tabular}{|cc|}
\hline Oído derecho & Oído izquierdo \\
\hline 21 & 22,5 \\
21,25 & 10 \\
32,5 & 21,25 \\
30 & 3,75 \\
26,25 & 26,25 \\
\hline
\end{tabular}

Valores en decibeles $(\mathrm{dB})$

Tabla 2. Medidas descriptivas/comparativas para el puntaje total y factores del cuestionario ABEL, tanto en normales como hipoacúsicos

\begin{tabular}{|llccccccc|}
\hline & Condición & Promedio & DE & Mediana & P25 & P75 & n & p value \\
\hline Puntaje total & Normales & 4,06 & 1,01 & 4,35 & 3,52 & 4,73 & 181 & 0,231 \\
& Hipoacúsicos & 3,86 & 0,84 & 3,93 & 3,52 & 4,26 & 14 & 0,208 \\
Oral-aural & Normales & 4,55 & 0,94 & 4,77 & 4 & 5,18 & 172 & 0,208 \\
Conciencia & Hipoacúsicos & 4,21 & 1,03 & 4,18 & 3,64 & 5,09 & 14 & 0,288 \\
auditiva & Normales & 4,34 & 1,02 & 4,67 & 3,67 & 5,11 & 173 & 0,283 \\
Aptitudes sociales & Hipoacúsicos & 4,22 & 0,75 & 4,5 & 4 & 4,67 & 14 & 0,123 \\
/conversación & Normales & 3,16 & 1,02 & 3,2 & 2,6 & 4 & 177 & 0 \\
\hline
\end{tabular}

DE: Desviación estándar. P25: Percentil 25. P75: Percentil 75. 
Anexo 1. Versión validada al español de cuestionario ABEL

\begin{tabular}{|c|c|}
\hline \multicolumn{2}{|r|}{ COMPORTAMIENTO AUDITIVO EN LA VIDA COTIDIANA (ABEL) } \\
\hline \multicolumn{2}{|c|}{ Nombre del niño: } \\
\hline \multicolumn{2}{|c|}{ Completado por: } \\
\hline \multicolumn{2}{|c|}{ Fecha: } \\
\hline \multicolumn{2}{|r|}{$\begin{array}{l}\text { INSTRUCCIONES: Nos gustaría saber cómo se siente acerca del desarrollo auditivo de su hijo. Por favor marque el número al lado de } \\
\text { cada elemento que mejor describa el comportamiento del niño durante la última semana. }\end{array}$} \\
\hline 0 & Nunca \\
\hline 1 & Casi nunca \\
\hline 2 & Ocasionalmente \\
\hline 3 & Cerca de la mitad del tiempo \\
\hline 4 & Frecuentemente \\
\hline 5 & Casi siempre \\
\hline 6 & Siempre \\
\hline 1. & Inicia conversaciones habladas con personas conocidas. \\
\hline 2. & Dice el nombre de una persona para obtener su atención. \\
\hline & Dice "por favor" o "gracias" sin que se le recuerde. \\
\hline & Responde verbalmente a los saludos de gente conocida. \\
\hline & Inicia conversaciones habladas con gente que no conoce. \\
\hline & Toma sus turnos en las conversaciones. \\
\hline 7. & Responde el teléfono apropiadamente. \\
\hline 8. & Responde cuando mencionan su nombre en la misma habitación. \\
\hline & Habla con un volumen de voz normal. \\
\hline & Pide ayuda en situaciones donde se necesita. \\
\hline & Hace ruidos inapropiados vocales. \\
\hline & Muestra interés en las conversaciones habladas alrededor de él/ella. \\
\hline & Responde verbalmente al saludo de una persona desconocida. \\
\hline 14. & Dice los nombres de sus hermanos, familiares, compañeros de clase. \\
\hline & Responde al timbre o golpes en la puerta. \\
\hline & Puede susurrar un mensaje personal. \\
\hline 17. & Se tranquiliza cuando se le pide. \\
\hline & Pregunta por sonidos que se escuchan en torno a él/ella (por ejemplo: aviones, camiones, animales). \\
\hline & Sabe cuando hacer sonidos fuertes (por ejemplo: portazos, pisadas de pie fuertes). \\
\hline 20. & Ignora el timbre del teléfono. \\
\hline 21. & Juega cooperativamente en grupos pequeños sin supervisión de un adulto. \\
\hline & (1) \\
\hline 23. & Experimenta con sonidos recién descubiertos. \\
\hline
\end{tabular}

distintos organismos, aún no existe un programa eficiente y amplio, que sea capaz de pesquisar y posibilite intervenir a niños hipoacúsicos a tiempo.

Los resultados de las características psicométricas de los instrumentos evaluados son favorables. Es ideal que la consistencia interna sea mayor a 0,7 , lo que se consigue en ambos cuestionarios. La confiabilidad test-retest, que hace mención a que el instrumento mida lo mismo en distintas situaciones, también fue favorable, siendo el ideal mayor a 0,7 . Por último, es relevante que 3 facto- res del test $A B E L$ den cuenta de gran parte de la varianza del instrumento $(80 \%)$, lo que guarda similitud con su versión original, asimilando ambas versiones del instrumento.

Sería de utilidad utilizar los cuestionarios propuestos (ABEL y TEAP) para el tamizaje de hipoacusia en escolares de nuestro país. Dichos cuestionarios demostraron tener una alta consistencia interna y confiabilidad para detectar niños con esta patología. Además, su estructura factorial, en que se identifican tres factores para el 
Anexo 2. Versión validada al español de cuestionario TEAP

EVALUACIÓN DE LOS PROFESORES DE RENDIMIENTO AUDITIVO (TEAP)

Por favor califique el comportamiento de este niño en comparación con otros niños de la misma edad y el contexto.

SECCIÓN A. OPCIONES DE RESPUESTA

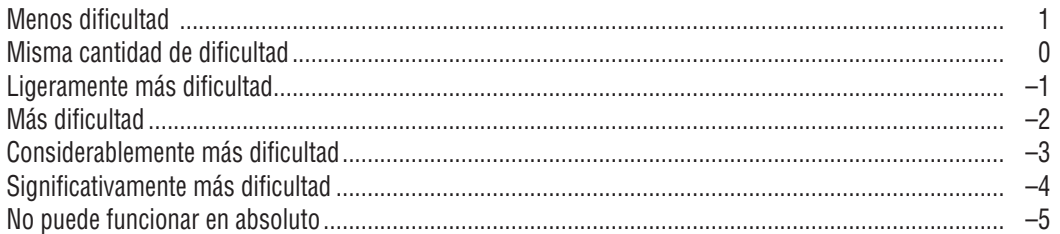

A1. Al escuchar en una habitación donde hay ruido de fondo (como otras personas hablando, niños jugando, etc.), este niño tiene dificultades para escuchar y entender.

A2. Al escuchar en una habitación en silencio (otros pueden estar presentes, pero están tranquilos), este niño tiene dificultades para escuchar y entender.

A3. Al escuchar en condiciones ideales (habitación tranquila, sin distracciones, cara a cara, con contacto visual), este niño tiene dificultad para oír y entender.

A4. Este niño tiene dificultad para seguir instrucciones orales de múltiples etapas.

\section{SECCIÓN B. Por favor marque en un círculo SI o NO}

B1. Este niño parece tener problemas para recoger información nueva hablada y puede requerir varias repeticiones para comprender el material.

B2. Este niño requiere con frecuencia señales visuales, además de la información auditiva, para ayudar a entender el plan de estudios.

B3. Este niño tiene dificultad para recordar la información auditiva, en comparación con otros niños.

B4. El niño muestra dificultad para formular o generar lenguaje expresivo, y/o muestra uso inapropiado del lenguaje. En caso afirmativo, por favor explique:

B5. El niño muestra problemas de lenguaje (se evidencia en el uso de los preguntas inapropiadas, pronombres, orden de las palabras, tiempos verbales).

En caso afirmativo, por favor explique:

B6. El niño muestra problemas de articulación consistentes en sustituciones, distorsiones u omisiones de los sonidos de las palabras (sobre todo con palabras que suenan similares).

En caso afirmativo, por favor explique:

caso de ABEL y uno para cada parte del TEAP, es semejante a la reportada para su versión original. Dichos factores explican un porcentaje importante de la varianza de la muestra. Hay que mencionar además que es la primera adaptación transcultural de instrumentos que permiten tamizar hipoacusia en escolares en nuestro medio, lo que permite ir sustentando programas de salud basados en evidencia.
En la presente muestra se encontró una prevalencia de hipoacusia del $5 \%$, lo que es cercano a lo reportado en un estudio realizado en EE.UU ${ }^{13}$ en que se observó el 7,6\% de problemas auditivos en niños entre los 6 y 11 años. Esto último orienta acerca del buen rendimiento de los cuestionarios utilizados para detectar hipoacusia en escolares de nuestro país. En el mismo sentido, las hipoacusias detectadas fueron todas leves; éstas junto a las 
moderadas son las más difíciles de identificar, ya que los niños no se dan cuenta hasta en $50 \%$ de los casos (14), probablemente por ser poco sintomáticas. A pesar de ello, nuestros tests fueron capaces de pesquisarlas.

\section{CONCLUSIÓN}

En conclusión, se consiguió realizar la adaptación transcultural de dos cuestionarios para tamizaje hipoacusia en niños. Estos resultados permitirían plantear la viabilidad de un programa formal de tamizaje de hipoacusia en escolares o preescolares en Chile basado en cuestionarios para hipoacusia.

\section{BIBLIOGRAFÍA}

1. Krauss M, Grucza R, Bierut L. Characterizing the Followers and Tweets of a Marijuana-Focused Twitter Handle. Journal of Medical Internet Research 2014; 16: 1.

2. White KR, Forsman I, Eichwald J, Munoz K. The evolution of early hearing detection and intervention programs in the United States. Seminars in Perinatology 2010; 34: 170-9.

3. Skarzynski H, Piotrowska A. Screening for pre-school and school-age hearing problems: European Consensus Statement. International Journal of Pediatric Otorhinolaryngology 2012; 76: 120-1.

4. Allan M, Le Roux G, Sonke Je, Piotrowska N, Streel M, Fagel N. Reconstructing historical atmospheric mercury deposition in Western Europe using: Misten peat bog cores, Belgium. The Science of the Total Environment 2013; 442: 290-301.

5. Benn A, Warburton D, Byrne JM, et al. A fetus with a chromosome 13 ring and placenta with chromosome $13 \mathrm{rod} /$ ring mosaicism. Prenatal Diagnosis 1983; 3: 297-302.

6. Bristow K, Fortnum H, Fonseca S, Bamford J. United Kingdom school-entry hearing screening: current practice. Archives of Disease in Childhood 2008; 93: 232-5.

7. Purdy SC, Farrington DR, Moran CA, Chard LL, Hodgson SA. A parental questionnaire to evaluate children's Auditory Behavior in Everyday Life (ABEL). American Journal of Audiology 2002; 11: 72-82.

8. Souza MR, Osborn E, GIL D, Iorio MC. Translation and adaptation of the ABEL: Auditory Behavior in Everyday Life questionnaire into Brazilian Portuguese. Jornal da Sociedade Brasileira de Fonoaudiologia 2011; 23: 368-75.

9. Purdy SC, Kelly AS, Davies MG. Auditory brainstem response, middle latency response, and late cortical evoked potentials in children with learning disabilities. Journal of the American Academy of Audiology 2002; 13: 367-82.

10. Guillemin F, Bombardier C, Beaton D. Crosscultural adaptation of health-related quality of life measures: literature review and proposed guidelines. Journal of Clinical Epidemiology 1993; 46: 1417-32.

11. Sininger YS, Doyle KJ, Moore JK. The case for early identification of hearing loss in children. Auditory system development, experimental auditory deprivation, and development of speech perception and hearing. Pediatric Clinics of North America 1999; 46: 1-14.

12. KeLMAN CA. Egocentric language in deaf children. American Annals of the Deaf 2001; 146: 276-9.

13. Niskar AS, Kieszak SM, Holmes A, Esteban E, Rubin C, BRoDY DJ. Prevalence of hearing loss among children 6 to 19 years of age: the Third National Health and Nutrition Examination Survey. JAMA: The Journal of the American Medical Association 1998; 279: 1071-5. 